\title{
Caracterização histopatológica de tumores mamários espontâneos de gatas (Felis catus) atendidas no Hospital Veterinário da UFRPE (Recife, Pernambuco, Brasil)
}

\author{
Histopathologic characterization of spontaneous mammary tumors \\ in cats (Felis catus) attended in Veterinary Hospital of UFRPE \\ (Recife, Pernambuco, Brazil)
}

\author{
Giuliana Viegas Schirato, ${ }^{*}$ Vandilson Rodrigues Silva, ${ }^{* *}$ Rodrigo Caldas Menezes, ${ }^{* *}$ Edlene Lima Ribeiro, ${ }^{* * *}$ \\ Christina Alves Peixoto, ${ }^{* * *}$ Catarina Oliveira Neves, ${ }^{* * * *}$ Mário Ribeiro Melo-Júnior, ${ }^{* * * *}$ Ana Lúcia Figueiredo Porto******
}

\begin{abstract}
Resumo
Os carcinomas mamários de gatas possuem prognóstico desfavorável, uma vez que tais tumores são bastante agressivos e com grande capacidade de gerar metástases. Objetivou-se, com este estudo, caracterizar sob o ponto de vista histopatológico, neoplasias mamárias de ocorrência espontânea em gatas (Felis catus). Trinta e cinco amostras de tecido neoplásico mamário de gatas foram obtidas por ressecção cirúrgica de animais oriundos da casuística do Hospital Veterinário da Universidade Federal Rural de Pernambuco (UFRPE), período de 2008 a 2010. Os cortes histológicos foram corados pela Hematoxilina-Eosina (HE) e as lâminas foram analisadas em microscopia de luz. Observou-se que 14,29\% das lesões eram benignas e $85,71 \%$ adenocarcinomas. As lesões de característica benigna foram classificadas como: $60 \%$ fibroadenomas, $20 \%$ como adenoma simples e $20 \%$ como adenose. Os adenocarcinomas apresentavam em sua maioria $(86,87 \%)$ presença de necrose e tal sinal esteve presente em grande parte do tecido neoplásico, enquanto apenas $40 \%$ das lesões benignas apresentavam pequenos focos de necrose. Em relação à presença de infiltrado inflamatório linfoplasmocitário, apenas os carcinomas apresentaram esse sinal. Comprovando a maior a agressividade dos tumores, foi observado um maior número de figuras de mitose em adenocarcinomas $(5,33 \pm 2,29$ mitoses por campo) quando comparadas às lesões de característica benigna $(0,8 \pm 0,45 / P=0,006)$. A maioria dos neoplasmas mamários de felinos apresenta característica de malignidade, gradação histológica alta, evidenciando um mau prognóstico para os indivíduos portadores.
\end{abstract}

Palavras-chave: neoplasias mamárias, caracterização histopatológica, carcinoma, Felis catus, tumores felinos.

\begin{abstract}
Breast carcinomas of cats have poor prognosis, since such tumors are very aggressive and with great ability to generate metastases. The aim of this study was to characterize the histopathological profiles, spontaneously occurring mammary tumors in cats (Felis catus). Thirty-five samples of breast tumor tissue of cats were obtained by surgical removal of animals from the series of the Veterinary Hospital of the Federal University of the state of Pernambuco (UFRPE), from 2008 to 2010. Sections were stained with hematoxylin-eosin (HE) and the slides were examined under light microscopy. It was observed that $14.29 \%$ of lesions were benign and $85.71 \%$ adenocarcinomas. The characteristic lesions were classified as benign, fibroadenomas $60 \%, 20 \%$ as a single adenoma and $20 \%$ as adenosis. The most of the cases of adenocarcinomas ( $86.87 \%)$ showed many areas of coagulative necrosis. While only $40 \%$ of the benign lesions showed small foci of necrosis. Regarding the presence of inflammatory infiltrate, only the carcinomas showed that signal. A greater number of mitotic figures were found in the adenocarcinomas than in the benign lesions $(5.33 \pm 2.29$ vs. $0.8 \pm 0.45$, respectively, $P=0.006)$. Most features of feline mammary neoplasms show histological characteristics of malignancy, indicating a poor prognosis for the affected individuals.
\end{abstract}

Keywords: breast cancer, histopathologic characterization, carcinoma, Felis catus, feline tumours.

\footnotetext{
* Programa de Pós-graduação em Ciência Veterinária, Departamento de Medicina Veterinária, Universidade Federal Rural de Pernambuco, Brasil. Centro de Experimentação Animal, Instituto Oswaldo Cruz, Fundação Oswaldo Cruz, Brasil.

** Hospital Veterinário, Departamento de Medicina Veterinária, Universidade Federal Rural de Pernambuco, Brasil.

*** Laboratório de Pesquisa Clínica em Dermatozoonoses, Instituto de Pesquisa Clínica Evandro Chagas, Fundação Oswaldo Cruz, Brasil.

**** Laboratório de Ultra-estrutura, Departamento de Entomologia, Instituto Aggeu Magalhães, Fundação Oswaldo Cruz, Brasil.

***** Departamento de Patologia, Universidade Federal de Pernambuco, Brasil.

****** Departamento de Morfologia e Fisiologia Animal, Universidade Federal Rural de Pernambuco, Brasil. Autor para correspondência: Universidade Federal Rural de Pernambuco, Departamento de Morfologia e Fisiologia Animal. Rua Dom Manoel de Medeiros, s/n, Dois Irmãos. Recife, PE - Brasil. CEP: 52171-900. Telefone: +55 81 33206391, Fax: +55 81 21268485. E-mail: analuporto@yahoo.com.br.
} 


\section{Introdução}

O carcinoma da glândula mamária da gata é o mais comum em qualquer outra espécie animal, com exceção do cão. Este tipo de tumor corresponde a $17 \%$ de todos os tumores que acometem a gata (Amorim et al., 2006; Magalhães et al., 2009). Os carcinomas mamários ocorrem em gatas mais velhas, entre oito e doze anos de idade, com média, dez anos. Não há predisposição racial estabelecida, embora alguns autores citem que em gatos siameses há o risco duas vezes maior do que todas as outras raças, além de desenvolver tumores mais precocemente (Amorim et al., 2006; Magalhães et al., 2009).

Os carcinomas mamários de gatas possuem diversos padrões histológicos, ou uma mistura deles em um mesmo tumor (Seixas et al., 2007). Tais tumores são compostos por células epiteliais luminais e ocorrência de carcinomas complexos é rara (Misdorp et al., 1999; Seixas et al., 2008). Macroscopicamente, possuem consistência firme e nodular, sendo simples ou múltiplos e as glândulas caudais dos animais são as mais acometidas. Pelo menos $25 \%$ das gatas afetadas possuem neoplasias ulcerativas (Magalhães et al., 2009).

A maioria dos tumores mamários de felinos é classificada como carcinomas simples, sendo que as apresentações histopatológicas mais frequentes são carcinomas tubulopapilares, sólidos e cribriformes. Sarcomas, carcinoma de células escamosas e carcinomas mucinosos são menos comuns (Millanta et al., 2002; Magalhães et al., 2009). Em função da característica de alta malignidade, o diagnóstico desta patologia deve ser realizado precocemente e de maneira precisa para que se inicie a terapia antitumoral o mais rápido possível, uma vez que tais condições influenciam diretamente no tempo de sobrevida do paciente (Giménez et al., 2010).

Objetivou-se com este estudo caracterizar, sob o ponto de vista histopatológico, neoplasias mamárias de ocorrência espontânea em gatas oriundas da casuística do Hospital Veterinário da Universidade Federal Rural de Pernambuco no período de 2008 a 2010.

\section{Materiais e métodos}

As amostras de tecido neoplásico mamário de gatas foram obtidas por ressecção cirúrgica (mastectomia simples ou dissecção em bloco) de animais oriundos da casuística do Hospital Veterinário da Universidade Federal Rural de Pernambuco (Recife, Pernambuco, Brasil), no período de 2008 a 2010. Nenhum animal incluído no estudo foi submetido à terapia antineoplásica prévia. Os 35 tumores coletados foram fixados em formalina 10\% preparada em tampão PBS 7,2 e, posteriormente, as amostras foram submetidas ao processamento histológico de rotina e incluídas em parafina.

Após microtomia, os cortes $(4 \mu \mathrm{m})$ foram corados pela Hematoxilina-Eosina (HE), para estudo histopatológico. Os cortes histológicos corados com HE foram analisados em microscopia de luz. A classificação tumoral foi realizada de acordo com a OMS (Misdorp et al., 1999), onde foram considerados aspectos histológicos mais frequentes neste tipo de lesão (presença de figuras de mitose aberrantes, necrose, calcificações, hemorragias e alterações vasculares).

A partir das análises histopatológicas dos tecidos foram determinados também o grau histológico $(\mathrm{H})$ e grau citológico (C), segundo a metodologia de Rosen e Oberman (1993). Para o grau histológico dos carcinomas mamários foi considerado o seguinte escore: $\mathrm{HI}=$ Carcinoma bem diferenciado; $\mathrm{HII}=$ Carcinoma moderadamente diferenciado; $\mathrm{HIII}=$ Carcinoma pouco diferenciado ou indiferenciado. Ao graduar os carcinomas em três grupos, são separados os tumores de possível comportamento biológico mais favorável $(\mathrm{HI})$ e os de provável prognóstico desfavorável (HIII) daqueles do grupo intermediário (HII), cujo comportamento é difícil de ser previsto. O grau citológico foi classificado em: $\mathrm{C} 1$ = carcinomas anaplásicos, com intensa atipia nuclear e numerosas mitoses; C2 = moderada atipia nuclear; C3 = leve atipia nuclear, raras.

Os resultados obtidos foram expressos como média \pm desviopadrão ( $\mathrm{M} \pm \mathrm{dp})$, submetidos ao teste Tukey, através do software PRISM 3.0, aceitando como estatisticamente significativos os valores comparados ao nível de significância de $5 \%$.

\section{Resultados e discussão}

Clinicamente, as lesões mamárias das gatas apresentavam-se como massas sólidas únicas ou múltiplas, com tamanho 2,16 $\pm 0,64 \mathrm{~cm}$ (lesões benignas) e 1,98 $\pm 0,73$ (carcinomas) e, em sua maioria, não ulceradas. Em relação à idade, as portadoras de lesões mamárias benignas possuíam 2,5 \pm 1,91 anos e as acometidas por carcinoma: 9,94 $\pm 2,26$ anos $(P=0,002)$.

Entre as 35 lesões mamárias de felinos estudadas, 14,29\% destas foram classificadas quanto ao critério biológico como benignas e $85,71 \%$ como malignas. Entre os tipos tumorais encontrados (Tabela 1), predominantemente, as lesões neoplásicas eram carcinomas sólidos em $51,43 \%$ dos casos, seguidos pelos carcinomas tubulopapilares (14,29\%) (Figura 1). Entre as lesões de característica benigna $(n=5)$, foram classificadas como: $60 \%$ fibroadenomas, $20 \%$ como adenoma simples e $20 \%$ como adenose.

Tabela 1: Distribuição das lesões mamárias espontâneas de gatas (Felis catus) oriundas da casuística do Hospital Veterinário da Universidade Federal Rural de Pernambuco (HV, DMV, UFRPE) no período de 2008 a 2010

\begin{tabular}{|c|c|}
\hline Classificação* & Percentual (\%) \\
\hline \multicolumn{2}{|l|}{ Lesões Benignas } \\
\hline Adenoma simples & $2,86 \%(1 / 35)$ \\
\hline Adenose & $2,86 \%(1 / 35)$ \\
\hline Fibroadenoma & $8,57 \%(3 / 35)$ \\
\hline \multicolumn{2}{|l|}{ Tumores Malignos } \\
\hline Carcinoma cribriforme & $2,86 \%(1 / 35)$ \\
\hline $\begin{array}{l}\text { Carcinoma em tumor benigno (adenoma } \\
\text { complexo) }\end{array}$ & $2,86 \%(1 / 35)$ \\
\hline Carcinoma mucinoso & $2,86 \%(1 / 35)$ \\
\hline Carcinoma sólido & $51,43 \%(18 / 35)$ \\
\hline Carcinoma sólido com áreas túbulo-papilares & $8,57 \%(3 / 35)$ \\
\hline Carcinoma túbulo-papilar & $14,29 \%(5 / 35)$ \\
\hline Carcinoma túbulo-papilar com áreas sólidas & $2,86 \%(1 / 35)$ \\
\hline
\end{tabular}

*Classificação segundo Misdorp et al. (1999). 

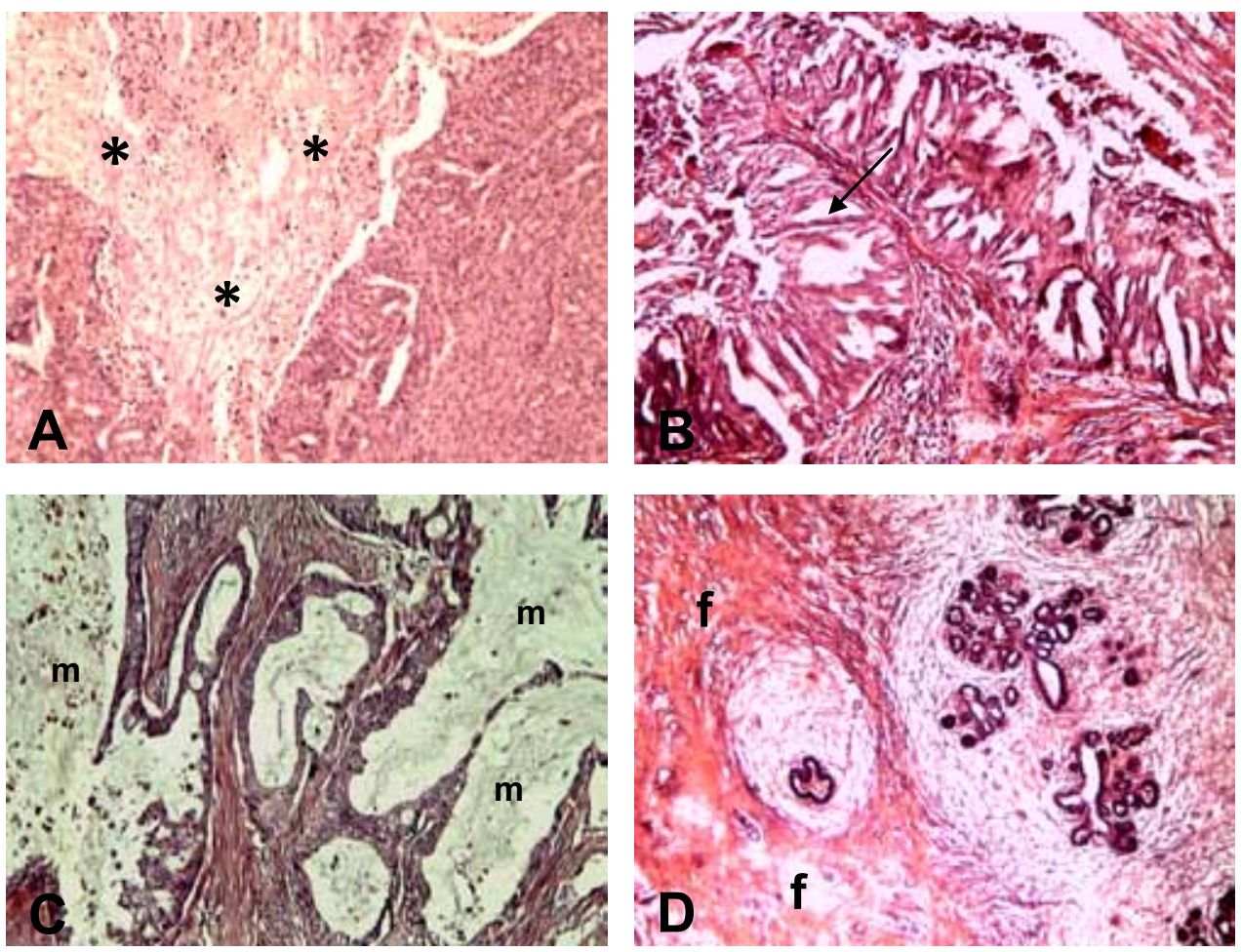

Figura 1: Apresentação histopatológica dos tipos tumorais dos neoplasmas mamários espontâneos de gatas (Felis catus) oriundas da casuística do Hospital Veterinário da Universidade Federal Rural de Pernambuco (HV, DMV, UFRPE) no período de 2008 a 2010. Hematoxilina-Eosina (HE). A (100X) Carcinoma sólido apresentando extensa área de necrose e atipia celular; B (100X) Carcinoma tubulopapilar; C (100X) Carcinoma mucinoso; D (100X) Fibroadenoma mamário. *- áreas de necrose; $\rightarrow$ - apresentação papilar; $m$ depósito de muco (glicosaminoglicanos); $\mathrm{f}$ - tecido fibroso.

Os tumores mamários de gatas (Felis catus) representam a terceira principal neoplasia nessa espécie, depois das neoplasias de pele e as de origem linfo-hematopoiética (Giménez et al., 2010). As lesões tumorais analisadas dos animais da casuística do Hospital Veterinário da UFRPE apresentaram percentual semelhante aos resultados de estudos anteriores, onde os carcinomas foram as neoplasias mais comuns, representando $80-90 \%$ dos casos (Amorim et al., 2006; Seixas et al., 2007). Uma vez que tais tumores são malignos e bastante agressivos, com alta capacidade de metástase e apresentam prognóstico ruim - sendo uma importante causa de mortalidade, principalmente em animais de meia-idade e idosos.

A avaliação histopatológica das lesões mamárias neoplásicas de gatas do presente estudo demonstrou que a maioria das neoplasias eram carcinomas simples de padrão histopatológico sólido. Também foram encontrados tumores com padrões histopatológicos diferentes na mesma neoplasia: carcinoma sólido com áreas tubulo-papilares, carcinoma tubulopapilar com áreas sólidas e carcinoma em tumor benigno (adenoma complexo). Tais resultados corroboram com dados da literatura, uma vez que nos tumores mamários de felinos, o tipo tumoral de maior prevalência é carcinoma simples, sendo que as apresentações histopatológicas encontradas são carcinomas tubulopapilares, sólidos e cribriformes (Millanta et al., 2002; Magalhães et al., 2009).

As lesões malignas analisadas eram multifocais, padrão de crescimento predominantemente invasivo, com presença marcante de anisocitose, necrose de moderada a intensa, figuras de mitose e infiltrado inflamatório linfoplamocitário. Entre tais parâmetros histopatológicos encontrados, os adenocarcinomas apresentavam em sua maioria $(86,87 \%)$ presença de necrose e tal sinal esteve presente em grande parte (superior a 30\%) do tecido neoplásico em $50 \%$ dos casos, enquanto $40 \%(n=2)$ das lesões benignas apresentavam poucos focos de necrose (Tabela 2).
Tabela 2: Distribuição dos parâmetros histopatológicos das lesões mamárias espontâneas de gatas (Felis catus) oriundas da casuística do Hospital Veterinário da Universidade Federal Rural de Pernambuco (HV, DMV, UFRPE) no período de 2008 a 2010

\begin{tabular}{l|c|c}
\hline & Lesões benignas & Carcinomas \\
\hline No. de casos & 5 & 30 \\
\hline
\end{tabular}

Necrose, $n(\%)$

\begin{tabular}{l|c|c}
\hline Ausente & $3(60)$ & $4(13,13)$ \\
+ & $2(40)$ & $6(20)$ \\
\hline++ & $0(0)$ & $5(16,67)$ \\
\hline+++ & $0(0)$ & $5(16,67)$ \\
\hline++++ & $0(0)$ & $10(33,33)$ \\
\hline
\end{tabular}

Infiltrado inflamatório, $n$ (\%)

\begin{tabular}{l|c|c}
\hline Ausente & $0(0)$ & $0(0)$ \\
\hline+ & $0(0)$ & $13(43,33)$ \\
\hline++ & $0(0)$ & $10(33,33)$ \\
\hline+++ & $0(0)$ & $7(23,33)$ \\
\hline
\end{tabular}

Invasão glandular, $n$ (\%)

\begin{tabular}{l|l|l}
\hline Presente & NA & $14(46,67)$ \\
\hline Ausente & NA & $16(53,33)$ \\
\hline
\end{tabular}

Necrose $-(+):<10 \% ;(++): 10-30 \% ;(+++): 30-50 \% ;(++++):>50 \%$. Infiltrado Inflamatório $-(+)$ : Leve; $(++)$ : Moderado; $(+++)$ : grave. NA - Não aplicável 
Em relação à presença de infiltrado inflamatório, nenhuma lesão benigna apresentou este sinal. No entanto, $100 \%$ dos carcinomas apresentavam infiltrado inflamatório (predominantemente formado por linfócitos e plasmócitos), classificado de moderado a intenso em $56,66 \%$ das amostras. As lesões mamárias malignas apresentavam invasão glandular em $46,67 \%$ dos casos.

A presença de figuras de mitose foi evidenciada tanto em lesões malignas quanto em lesões benignas; no entanto, visualizada em maior número em adenocarcinomas $(5,33 \pm 2,29$ mitoses por campo) e 0,8 $\pm 0,45$ em lesões de característica benigna $(P=0,006)$.

Neoplasias epiteliais malignas originam-se de células epiteliais dos ductos mamários maiores, ductos interlobulares, dúctulos intralobulares e epitélio secretório dos ácinos (alvéolos). Em geral, ocorrem três padrões de proliferação (acinar, tubular e papilar), mas em muitos casos pode-se observar mais de um padrão em diferentes regiões da neoplasia. Cada tipo também pode estar acompanhado por uma proliferação concomitante de células mioepiteliais circundadas por uma matriz condromucinosa (Jones et al., 2000). Em cadelas, os carcinomas mamários apresentam células neoplásicas apresentando pleomorfismo, anisocariose e uma elevada taxa de índice mitótico ( $>4$ mitoses por ampliação de 400X), com áreas multifocais de necrose (Klopjleisch et al., 2010).

O grau histológico $(\mathrm{H})$ e citológico $(\mathrm{C})$ dos adenocarcinomas também foi avaliado. Em relação ao grau histológico, 3,33\% (1/30) foram classificadas como HI, 46,67\% (14/30) como HII e $50 \%(15 / 30)$ como HIII. Ao graduar os carcinomas em três grupos, são separados os tumores de possível comportamento biológico mais favorável $(\mathrm{HI})$ e os de provável prognóstico desfavorável (HIII) daqueles do grupo intermediário (HII), cujo comportamento é difícil de ser previsto.

A gradação histológica é um bom parâmetro para estratificar os tumores de acordo com sua agressividade biológica (Karayannopoulou et al., 2005). Dessa forma, a utilização desse método de gradação é uma ferramenta útil no diagnóstico de neoplasia mamária de felinos, favorecendo consequentemente o prognóstico de sobrevida dos animais. Em humanos, a combinação do tipo e grau histológicos fornece uma avaliação mais acurada do prognóstico do câncer de mama, quando comparada com a avaliação histopatológica com único meio de diagnóstico. O grau histológico também pode fornecer informações úteis para prever a resposta à quimioterapia e, portanto, ser um fator preditivo (Ellis et al., 2003).

Seixas e colaboradores (2011) verificaram que HIII foi o grau histológico mais prevalente em neoplasias mamárias de felinos. Tal grau, segundo os autores, é associado a um pior prognóstico, resultando menor tempo de sobrevida dos animais após a remoção cirúrgica dos tumores. Tais resultados corroboram com os encontrados no presente estudo, uma vez que $50 \%$ das neoplasias mamárias das gatas foram classificadas no grau HIII.

O grau citológico (C), também denominado de "grau de diferenciação nuclear", é uma análise que leva em conta a morfologia nuclear da célula neoplásica, representando um importante parâmetro prognóstico para carcinomas mamários em humanos e animais (De Vico e Maiolino, 1997; Simeonov e Simeonova, 2009). Tal sistema de avaliação é o oposto do grau histológico, ou seja: C1 representa pior prognóstico e C3 melhor prognóstico quando comparado às demais classificações.

Castagnaro e colaboradores (1998) ao caracterizarem neoplasias mamárias de gatas sob o ponto de vista citológico constataram que tais lesões apresentavam células com núcleos hipercromáticos e presença de figuras de mitose. Amorim e coautores (2006), além de tais características, verificaram que no tecido neoplásico foram encontradas células e núcleos com variação marcante de tamanho, presença de nucléolo e pleomorfismo celular. Já as lesões da casuística de nosso estudo, em relação ao grau citológico: 23,33\% (7/30) das neoplasias malignas apresentavam grau C1 (apresentando-se como carcinomas anaplásicos, com intensa atipia nuclear e numerosas mitoses) e 76,67\% (23/30) foram classificadas como $\mathrm{C} 2$, sendo caracterizadas por moderada atipia nuclear.

\section{Conclusão}

A maioria dos neoplasmas felinos apresenta característica de malignidade (sendo o carcinoma sólido o tipo tumoral mais frequente) e o tipo tumoral benigno mais encontrado é o fibroadenoma. Histologicamente, os tumores malignos são multifocais, padrão de crescimento predominantemente invasivo, com presença de necrose, figuras de mitose, infiltrado inflamatório e gradação histológica (HII e HIII) e citológica (C1 e C2) alta. Tais características propiciam um mau prognóstico para os indivíduos portadores e, consequentemente, resultar em um menor tempo de vida pós-cirúrgico aos animais acometidos.

\section{Agradecimentos}

Agradecemos a Vera Lúcia Farias Cavalcanti e Gustavo Andrey Lins Falcão de Alcântara, pelo suporte técnico realizado.

\section{Referências}

AMORIM, F.V.; SOUZA, H.J.M.; FERREIRA, A.M.R et al. Clinical, citological and histopathological evaluation of mammary masses in cats from Rio de Janeiro, Brazil. J. Feline Med. Surg., v. 8, p. 378-388, 2006.

CASTAGNARO, M.; DE MARIA, R.; BOZZETA, E. et al. Ki-67 index as indicator of the post-surgical prognosis in feline mammary carcinomas. Res. Vet. Sci., v. 65, p. 223-226, 1998.

DE VICO, G.; MAIOLINO, P. Prognostic value of nuclear morphometry in feline mammary carcinomas. J. Comp. Pathol., v. 117, p. 99-105, 1997.
ELLIS, I. O.; SCHMITT, S.J.; SASTRE-GARAU, X. et al. Invasive breast carcinoma. In: TAVASSOLI, F.A.; DEVILLE, P. (Eds.). Pathology and genetics of tumours of the breast and female genital organs. World Health Organization Classification of Tumours. Lyon: IARC Press, 2003, p. 13-59.

GIMÉNEZ, F.; HECHT, S.; CRAIG, L.E. et al. Early detection, aggressive therapy: optimizing the management of feline mammary masses. J. Feline Med. Surg., v. 12, p. 214-224, 2010.

JONES, T.C.; HUNT, R.D.; KING, N.W. Patologia veterinária. 6. ed. São Paulo: Manole, 2000. 1415 p. 
KARAYANNOPOULOU, M.; KALDRYMIDOU, E.; CONSTANTINIDIS, T.C. et al. Histological grading and prognosis in dogs with mammary carcinomas: application of a human grading method. J. Comp. Pathol., v. 133, p. 246-252, 2005.

KLOPJLEISCH, R.; SHÜTZE, M.; GRUBER, A.D. Loss of p27 expression in canine mammary tumors and their metástases. Res. Vet. Sci., v. 88, n. 2, 2010.

MAGALHÃES, M.; OLIVEIRA, F.S.; HAKATA, A. et al. Neoplasmas mamários em gatas - revisão de literatura. Rev. Clin. Vet., v. 79, p. 48-52, 2009.

MILLANTA, F.; LAZZERI, G.; MAZZEI, M. et al. MIB-1 labeling index in feline dysplastic and neoplasic mammary lesions and its relationship with postsurgical prognosis. Vet. Pathol., v. 36, p. 619636, 2002.

MISDORP, W.; ELSE, R.W.; HELLMAN, E. et al. Histological classification of the mammary tumors of the dog and the cat World Health Organization International Histological Classification of Tumors of Domestic Animals. 2. ed. Washington, D.C: Armed Force Institute of Pathology, 1999. $57 \mathrm{p}$.
ROSEN, P.P.; OBERMAN, H.A. Tumor of the mammary gland. In: Atlas of tumor pathology. 3. ed. Washington, D.C.: Armed Forces Institute of Pathology, 1993. p. 78-87.

SEIXAS, F.; PALMEIRA, C.; PIRES, M.A. et al. Grade is an independent prognostic factor for feline mammary carcinomas: $A$ clinicopathological and survival analysis. Vet. J., v. 187, n. 1, p. 65-71, 2011.

SEIXAS, F.; PALMEIRA, C.; PIRES, M.A. et al. Are complex carcinoma of the feline mammary gland and other invasive mammary carcinoma identical tumours? Comparison of clinicopathologic features, DNA ploidy and follow up. Res. Vet. Sci., v. 84, p. 428-433, 2008.

SEIXAS, F.; PALMEIRA, C.; PIRES, M.A. et al. Mammary invasive micropapillary carcinoma in cats; clinicopathologic features and nuclear DNA content. Vet. Pathol., v. 44, p. 842-848, 2007.

SIMEONOV, R.; SIMEONOVA, G. Nuclear cytomorphometry in feline mammary gland epithelial tumours. Vet. J., v. 179, p. 296$300,2009$. 